\title{
Opciones clave para una propuesta de inclusión educativa
}

\author{
Juan Carlos Jiménez Torres ${ }^{1}$ \\ juan.jimenez@feyalegria.org.ec
}

\section{Resumen (Analítico):}

En la tarea de inclusión educativa necesitamos tomar determinadas opciones clave para que se lleve a cabo de forma adecuada. Presentamos estas opciones importantes: Optamos por el Enfoque de Atención a la Diversidad, fundamentada en los derechos humanos, y por el Diseño Universal de Aprendizaje, (DUA). Optamos por el Enfoque Ecológico Funcional, (EEF) que es el que mejor está respondiendo a las necesidades de los estudiantes con diversidad funcional. Optamos por una Inclusión Temprana en el Sistema Educativo. Optamos por una inclusión por Tránsito Educativo, con sus ritmos y tiempos adecuados al crecimiento. Optamos por diferentes caminos de inclusión, en la educación ordinaria y también en educación especializada. Optamos por una inclusión educativa con calidad y recursos suficientes que incluya también la necesaria Transición a la Vida Adulta y Laboral.

Palabras clave: Atención a la Diversidad. Enfoque Ecológico Funcional. Inclusión Temprana. Inclusión por Tránsito Educativo. Transición a la Vida Adulta y Laboral.

\section{Key options for an educational inclusion proposal}

\section{Abstract (Analytical):}

In the task of educational inclusion, we need to take certain key options

1 Máster en Ciencias de la Educación Religiosa por la Universidad Pontificia de Comillas. Diplomado en Filosofía y Letras por la Universidad Complutense de Madrid. Cursos de postgrado en Educación Especial e Inclusiva por la Universidad Politécnica Salesiana y Universidad Católica Andrés Bello. Acompañante de Inclusión en Fe y Alegría Zona Santo Domingo, Ecuador. 
to ensure they are carried out properly. We go over these fundamental options: We choose to focus on the Attention to Diversity approach, based on human rights, and the Universal Design of Learning (UDL). We opt for the Functional Ecological Approach, (FEA) which is the one that is responding more to the needs of students with functional diversity. We opt for an inclusion in the education system from an early age. We opt for the Inclusion in their Educational Transition for the growth period, with its proper rhythms and times. We opt for different paths of inclusion, as in ordinary as in specialist education. We opt for an educational inclusion with quality and adequate resources that provide also the transition needed towards adulthood and working life.

Keywords: Attention to Diversity. Functional Ecological Approach. inclusion in the education system from an early age. Inclusion in the educational transition. Transition towards adulthood and working life.

\section{Introducción:}

Realizar una propuesta general de inclusión, desde edades tempranas, es algo muy grande y quizás muy ambicioso; pero la realidad es que los estudiantes que necesitan la propuesta son de todas las edades y niveles educativos, y la mayoría "ya los tenemos en casa". Otros, están llamando a nuestras puertas. Con lo cual, a una institución educativa no le queda más remedio que dar respuesta a esta necesidad real.

Esta reflexión nace de la experiencia de varios centros de Fe y Alegría Ecuador, que efectivamente quisieron dar respuesta a las necesidades que se les planteaban. Esto obligó a formarse, a investigar, a poner en práctica y a volver a investigar conjuntamente para ser lo más certeros posibles. Aun así tenemos la convicción de que no es una tarea acabada y que hay que seguir reflexionando y construyendo.

La tesis que planteamos es sencilla de formular y compleja en el desarrollo: Podemos afirmar que la tarea de la inclusión educativa no se puede realizar de cualquier manera ni de forma apresurada, y que necesitamos tomar determinadas opciones clave para que se lleve a cabo de forma adecuada. Ahora bien, las opciones son muy amplias 
para ser desarrolladas en un solo artículo. Sin embargo, creemos necesario presentarlas todas ellas para garantizar una propuesta de inclusión completa y abarcadora, donde se presente una buena fundamentación, y también caminos y propuestas de realización.

Los objetivos de la investigación por tanto van en estas direcciones, y podemos formularlos así: Primero, fundamentar la propuesta con dos enfoques cruciales para la inclusión, como son el enfoque de Atención a la Diversidad y el Ecológico Funcional. Segundo, ofrecer caminos de inclusión, desde edades tempranas, con los ritmos y tiempos adecuados al crecimiento. Y por último añadir al proceso la necesaria transición a la vida adulta y laboral.

Vamos a ir desarrollando las opciones clave, empezando por las más generales, y al final haremos algunas conclusiones y plantearemos retos importantes.

\section{I.- Nuestra primera gran opción: \\ El enfoque de "Atención a la Diversidad" fundamentada en los Derechos Humanos.}

Este es un enfoque y estilo de inclusión muy atrayente que desplazaría las corrientes integradoras y nos hace repensar las hasta hoy llamadas Adaptaciones Curriculares. Todavía se sigue con el modelo integrador, buscando atender las limitaciones de ciertos estudiantes con algún tipo de merma en el aprendizaje, y no se tiene la mirada amplia de atender la diversidad del grupo, o de optimizar los recursos y potencialidades de cada estudiante (García-García et al., 2013).

La atención a la diversidad se concibe como el conjunto de ac- 
ciones educativas que en un sentido amplio intentan prevenir y dar respuesta a las necesidades, temporales o permanentes, de todo el alumnado del centro (Educacantabria 2017). Desde esta perspectiva creemos que es necesario rebasar el modelo clínico y optar por un enfoque de derechos que resalta las fortalezas y potencialidades de las personas poniendo a un lado las debilidades ${ }^{3}$.

Este es un gran reto específicamente en Ecuador donde tenemos lineamientos y guías para atender a la inclusión basados en Adaptaciones Curriculares. En realidad, podemos decir que en este proceso de adecuaciones del currículo, si se hace bien, se puede atender a la diversidad, porque se contemplan las adaptaciones grupales, de aula, y de grado uno y dos, (esto es, de acceso y de metodologías), que buscan atender a la diversidad de todos y cada uno. Los documentos oficiales de inclusión ya quieren expresarse en términos de diversidad, incluso en sus eslóganes principales. "Somos diversos, somos inclusivos" reza la portada de uno de los documentos que quiere trabajar la atención a las necesidades educativas diversas y para ello plantea una Guía de adaptaciones curriculares (MINEDUC 2013). Por tanto es una corriente o enfoque en el que tenemos que seguir indagando y aprendiendo. Lo presentamos de una forma general.

Desde el Diseño Universal de Aprendizaje, DUA. Una posible respuesta de atención a la diversidad la encontramos en el denominado Diseño Universal para el Aprendizaje (DUA), desarrollado por el Center for Applied Special Technology (Centro de Tecnología Especial Aplicada, CAST).

La propuesta del CAST pasa por dotar de mayor flexibilidad al currículo, a los medios y a los materiales, de modo que todo el alumnado pueda acceder al aprendizaje.

- En la base del DUA están los tres principios fundamentales, que corresponden a las tres redes cerebrales implicadas en el aprendizaje (redes de reconocimiento, redes estratégicas y redes afectivas). Estos

3 Por eso optamos por el término de "Diversidad Funcional", que, como bien se desarrolla en el artículo "Diversidad funcional, nuevo término para la lucha por la dignidad en la diversidad del ser humano", apunta a que la sociedad se vaya sensibilizando y se promueva un término que aporte una nueva visión social respecto a la discapacidad (Javier Romañach, Manuel Lobato, 2005). 
principios se han convertido en un referente obligado que aparece en la mayoría de la bibliografía sobre el tema. Son los siguientes:

1.- Multiplicidad de medios de representación con el fin de dar a los alumnos diferentes opciones para la adquisición de información y la construcción del conocimiento.

2.- Multiplicidad de medios de acción y de expresión, para proporcionar opciones a los alumnos para demostrar sus conocimientos.

3.- Multiplicidad de medios de compromiso, para aprovechar los intereses y la motivación de los alumnos ${ }^{4}$.

Los tres principios del DUA indican que es necesario que los docentes ofrezcan al alumnado un amplio rango de opciones para acceder al aprendizaje. Los puedo tener en cuenta para todos los elementos del currículo.

- Un buen punto de partida puede ser el siguiente: ¿Cómo las actividades que planifico dan cabida a todos los estudiantes? Este ejercicio de planificación permite encontrar varias actividades para un mismo contenido; para lo cual es importante conocer los estilos y ritmos de aprendizaje de cada estudiante. Esto se logra observando su desempeño en clase, proceso de aprendizaje y motivaciones. En algún momento tengo que averiguar:

¿Cómo les gusta aprender, qué actividades les gusta hacer con mayor frecuencia, qué actividades pueden mejorar su aprendizaje? La flexibilidad del currículo permite hacer ajustes necesarios para responder al desafío de la diversidad. O dicho de otro modo: ¿Cómo aprenden mejor mis estudiantes? ¿Cuáles son sus niveles de aprendizaje? ¿Tengo estudiantes con necesidades diversas de aprendizaje?

En el fondo, con este enfoque se trata de centrarnos, no tanto en 
la necesidad educativa de un estudiante en particular, sino en la diversidad del grupo.

- Atención a las barreras de aprendizaje: El Diseño Universal de Aprendizaje nos advierte de la necesidad de reparar en las barreras de aprendizaje ${ }^{5}$. No se trata de ver las dificultades del estudiante, que es lo que hemos hecho casi siempre, sino cuáles son las barreras que impiden su aprendizaje.

Las barreras constituyen un impedimento, traba u obstáculo que limita o impide la participación e inclusión del sujeto en su contexto social. (Gallegos y De la Cadena, 2014). En nuestro caso todas aquellas restricciones que se presentan en el contexto educativo. Hay barreras de muchos tipos, pero giran en torno a estos tres ámbitos: 1. Barreras de acceso. 2. Barreras psicopedagógicas o curriculares. 3. Barreras actitudinales o de relación.

- Conocimiento de cada persona con diversidad funcional. Cada persona tiene sus propias características, modos de aprender y aprehender el entorno. Por tanto, es indispensable conocer y valorar las potencialidades y habilidades que tiene el estudiante y así determinar los apoyos que requiere en función de sus necesidades. Es necesario poner mucha atención a las barreras para el aprendizaje (pedagógicas-curriculares) y qué acciones inclusivas debo poner en marcha para superar dichas barreras. Contemplar la posibilidad del uso de las TICs como un medio privilegiado y transversal para la atención a la diversidad...entre otras. Todo ello siempre en colaboración con los docentes, estudiantes y padres de familia.

- Equipo de apoyo: docente, familia, apoyo profesional. En este modelo el apoyo no sólo es para los estudiantes con diversidad funcional, sino, sobre todo, el apoyo es al docente que realiza la planificación de unidades didácticas para todos. Apoyo al docente en la elaboración de recursos didácticos y metodologías que no sólo ayudan al estudiante con diversidad funcional sino a todos los estudiantes. Tanto el docente de aula, como el docente de apoyo, trabajan juntos. 


\section{II.- Nuestra segunda gran opción: El Enfoque Ecológico Funcional, (EEF).}

Casi todas las propuestas de educación especial e inclusiva en la actualidad están de acuerdo en que es un enfoque adecuado, aunque algunos lo siguen y aplican de una manera más purista o detallada que otros. En casi todos los foros, encuentros, cursos y congresos lo hemos percibido así. A su vez este enfoque recibe y comparte corrientes y propuestas de grandes autores. Podemos nombrar las siguientes teorías: la socio-histórica-cultural de Vygotsky, el aprendizaje significativo de Ausubel, las inteligencias múltiples de Gardner o el aprendizaje por descubrimiento de Bruner'.

Este modelo de currículo funcional se basa en la enseñanza de destrezas/habilidades que se enseñan en un contexto natural tomando en cuenta lo que sabe el estudiante y lo que va a necesitar para su futuro. Ellos proponen tomar en cuenta la funcionalidad del estudiante y su edad cronológica, y no el cociente intelectual ni edad mental. En su día empezó llamándose incluso "Currículo" Ecológico Funcional. Muchas de sus propuestas siguen hoy en vigor, pero dado que hay un solo $\mathrm{Cu}$ rrículo Nacional, se prefiere llamar "Enfoque".

Ofrecemos una presentación de los elementos importantes de este enfoque, que hemos ido recogiendo en diferentes cursos, el más importante fue el "Programa de capacitación a docentes y familias para el desarrollo de la Educación Especial" en los años 2011 y 2012, preparado por la Universidad Politécnica Salesiana de Quito, coordinado por Myriam Gallegos, María Graciela Laynes, Melva Yépez, Rocío Medina y Luz Elena Tirado.

Los objetivos de este enfoque los podemos resumir así:

- Brindar enseñanzas funcionales significativas.

- Respetar la edad cronológica.

6 Por fortuna, estas últimas corrientes de pedagogía están ya presentes en las reformas curriculares oficiales, y ya las han incorporado en la fundamentación y en la base teórica de sus documentos (Ej. Currículo oficial de Educación Inicial del MINEDUC en 2013). 
- Las enseñanzas deben hacerse en contextos naturales.

- Respetar sus necesidades individuales.

- Tomar en cuenta las demandas de la vida adulta.

- Propiciar la interacción y la autodeterminación.

- Propiciar la inclusión en el hogar-escuela-comunidad.

\section{Dominios Ecológicos}

En el Enfoque Ecológico Funcional se habla de Dominios Ecológicos, que los podemos definir como los ámbitos que todos los seres humanos deben dominar en la vida para desarrollar su autonomía, y así poder desenvolverse adecuadamente en sociedad. Es interesante comprobar cómo hay una similitud entre los diferentes dominios del enfoque ecológico y los ejes y ámbitos de desarrollo y aprendizaje del currículo de Educación Inicial del MINEDUC. Los define como "campos generales de desarrollo y aprendizaje que responde a la formación integral y orientan las diferentes oportunidades de aprendizaje" (MINEDUC 2014). De esta manera se propone también un desarrollo integral en todos los aspectos, promoviendo oportunidades variadas de aprendizaje, estimulando la exploración en ambientes ricos y diversos...etc. Vamos a exponer los dominios y haremos una relación con los ejes y ámbitos de desarrollo y aprendizaje del MINEDUC. Ambos nos han ayudado a organizar los aprendizajes de nuestros estudiantes desde edades tempranas. Exponemos de forma resumida los dominios:

1.- DOMINIO DOMÉSTICO: se refiere a la higiene personal, ropa y vestido, alimentación y cocina, limpieza y aseo... No es difícil ver la correspondencia con el desarrollo de las destrezas del ámbito de Identidad y autonomía, y también con destrezas de Relación con el medio natural.

2.- DOMINIO COMUNITARIO: Desde las instalaciones de la escuela o colegio, señales de tránsito, orientación, transporte y movilización en el barrio o ciudad, visita a lugares estratégicos y necesarios de su entorno. Aquí vemos la correspondencia con el ámbito de Relación con el medio natural y cultural, y con el ámbito de Convivencia.

3.- DOMINIO RECREATIVO: Todo lo referente a la danza, música, teatro, deportes, juegos populares, excursiones y paseos, visitas a 
espacios culturales y recreacionales...La correspondencia sería con los ámbitos de Relaciones con el medio natural y cultural, Expresión corporal y motricidad y Expresión artística.

4.- DOMINIO VOCACIONAL para prepararlos a la transición a la vida adulta y laboral: Habilidades y competencias para el trabajo, elaboración del perfil vocacional, visitas prelabolares, prácticas laborales, talleres de formación profesional, ubicación y seguimiento laboral. Encontramos correspondencia con los ámbitos de Identidad y autonomía, Convivencia y Relación con el medio natural y social.

5.- DOMINIO ACADÉMICO FUNCIONAL: Comunicación y lenguaje, pre-escritura, escritura funcional, cálculo elemental y funcional, funciones cognitivas, como memoria, conceptos básicos de tamaño, forma, discriminación, razonamiento...

En este dominio encontramos correspondencia directa con el ámbito de Relaciones lógico-matemáticas y con el de Comprensión y expresión del lenguaje. E indirectamente con todos los demás ámbitos en los diferentes ambientes y sub-ambientes en los que se desarrollan las actividades funcionales.

Organización de los grupos. Además de los dominios, el Enfoque Ecológico organiza los grados por el factor de edad cronológica: Supone tener en cuenta más la edad que los conocimientos, ya que el desarrollo y madurez personal de un adolescente es el de su edad cronológica. Con eso evitamos que un estudiante de 14 años que es ya un adolescente, esté con niños de 8 años. Aun así para organizar los grupos o incluirlos en un grado determinado tenemos en cuenta un rango de más menos 2 años.

Planificación centrada en la persona. Otro elemento clave que va a estar presente siempre es la planificación centrada en la persona. Lo realizamos mediante la elaboración de los Planes de Acción (Making Actions Plans), comúnmente llamados "Mapeos". Para ello compartimos con la familia y el estudiante sus sueños, fortalezas, gustos... y realizamos las valoraciones funcionales personalizadas. Se trata de encontrar un plan de acción para su vida, a corto, mediano o largo plazo, y no 
tanto de buscar el cociente intelectual, que muchas veces ha etiquetado a la persona y no nos ha guiado en su desarrollo.

La familia con el equipo colaborativo. Otro elemento clave es la incorporación más explícita de la familia en el proceso educativo: desde el mapeo y la valoración funcional del principio, planificando y proponiendo actividades diarias, revisando actividades, y también en las valoraciones parciales, quimestrales... Se trata de que forme parte del equipo colaborativo junto con los profesionales que atendemos a los estudiantes (profesores, psicopedagogos, logopedas...) No se trata de trabajar aisladamente, sino que nos vemos como un equipo: Ya no están solamente en su despacho, sino que rotan también por las aulas para compartir y apoyar a docentes y estudiantes.

Actividades funcionales. La realización de actividades funcionales en ambientes naturales es una pieza fundamental del EEF, que entiende el aprendizaje no como una asimilación pasiva de información, sino como una práctica directa de las habilidades y destrezas que necesita, y además en su lugar natural. Por ejemplo: ir al mercado, a una tienda, a la frutería...cruzar el semáforo, subir al bus...etc. Y mientras realizamos la actividad podemos desarrollar destrezas de todo tipo: destrezas lógico-matemáticas, cuando compro y me dan el cambio, de lengua y comunicación, cuando hago la lista de las frutas que quiero comprar...etc.

En resumen, podemos afirmar que, con la experiencia y conocimiento de los nuevos paradigmas de la educación, este enfoque sí da la oportunidad de desarrollar los objetivos académicos funcionales a toda la población con diversidad funcional de la escuela especializada o de los que estén incluidos en la escuela ordinaria. Pensamos que puede ofrecer a nuestros estudiantes enseñanzas significativas, integradas en las diferentes áreas de desarrollo y relacionadas con los diferentes ambientes. A la par, les damos oportunidad de resolver los retos de su vida diaria, pueden crecer en autonomía y en inclusión social y laboral. 


\section{III.- Nuestra tercera gran opción: "Inclusión Temprana" en el Sistema Educativo.}

Pensamos que el mejor proceso de inclusión debe empezar lo antes posible, desde Educación Inicial 1, frente a la inclusión tardía que ya hemos vivido y que sabemos que tiene muchas limitaciones. Hay múltiples documentos que justifican esta opción, entre ellos el último currículo de Educación Inicial del Ministerio de Educación de Ecuador, que propone empezar el proceso educativo desde Educación Inicial 1 (0-3 años), pasar a Educación Inicial 2 (4 años), Primero de EGB 5 (años)...etc. También lo justifica la praxis de la mayoría de los países que tienen esa inclusión en edades tempranas.

En realidad todos estamos de acuerdo en que garantizar experiencias positivas durante los primeros años de vida -desde el entorno familiar estimulante y lleno de afecto hasta una educación de calidad con un entorno lúdico y adecuado cuidado de salud y nutrición- pueden potenciar todos los ámbitos del desarrollo infantil, y puede tener incidencia a lo largo de la vida de la persona.

Por otro lado, El Laboratorio de Investigación e Innovación en Educación para América Latina y el Caribe, SUMA, nos muestra el impacto y evidencia considerable de la intervención educativa en la primera infancia. En concreto en el tema específico de la diversidad funcional, la OMS y UNICEF publicaron en el año 2013 un documento a debate titulado "El desarrollo del niño en la primera infancia y la discapacidad" donde se afirma:

"El propósito de este documento es ofrecer un panorama de la discapacidad en la primera infancia y destacar la importancia de brindarles oportunidades a los niños con discapacidad en este período, para que tengan las mismas oportunidades que los demás niños de desarrollar todo su potencial y participar de manera significativa en sus hogares, escuelas y comunidades." (OMS \& UNICEF, 2013 PAG). 
Por lo tanto, esta opción contempla ofrecer las mismas oportunidades de desarrollar todo el potencial que tienen las personas con alguna diversidad funcional, de manera que se oferten grados desde Educación Inicial I especializada, Educación Inicial II, $1^{\circ}$ de EGB...etc. Estos grupos siguen muy de cerca el Enfoque Ecológico Funcional, con una planificación centrada en la persona.

\section{IV.- Cuarta opción: Inclusión por "tránsito educativo".}

Inclusión progresiva. Pensamos que la inclusión no se pueda dar "de golpe", sino paso a paso, con sus ritmos y tiempos adecuados al crecimiento y desarrollo de los estudiantes. De esta manera los estudiantes "transitan" de un grado a otro por el camino educativo más adecuado. Esta idea complementa la opción anterior, para que la inclusión se cumpla de manera progresiva y procesual. Se justifica también con el currículo del Ministerio de Educación, y tiene como base teórica las propuestas de Vygotsky de la Zona de Desarrollo Próximo y el aprendizaje significativo de Ausubel, que sostienen que el aprendizaje se produce desde lo que ya conocen. Por supuesto las aportaciones de la neurociencia nos dicen que hay períodos más sensibles para que se produzcan la modificación y ampliación neuronal (neuroplasticidad), y los primeros años es una de ellas. En último término sabemos que el desarrollo infantil es "un proceso de cambios continuos por el que atraviesan los niños y niñas desde su concepción, que, en condiciones normales, garantizan el crecimiento, la maduración y la adquisición progresiva de las complejas funciones humanas como el habla, la escritura, el pensamiento, los afectos, la creatividad..."(MIES-INFA, 2011).

Parece lógico pues, que si se propone un sistema educativo desde edades tempranas y de forma procesual para los estudiantes de educación ordinaria, también puede ser el mejor momento y la mejor forma para la inclusión educativa de personas con diversidad funcional. 
Transición de la educación especial a la ordinaria. Por otro lado, otra acepción de "transición" resulta de la necesaria transición de algunos estudiantes de la educación especial a la ordinaria, sobre todo en los primeros años de Educación Inicial y Preparatoria. Pensamos que hay un tiempo privilegiado hasta los cinco años para desarrollar las potencialidades en grupos más pequeños y así tomar la decisión de transición a la educación ordinaria. En ocasiones, la experiencia nos dice que no ha habido más remedio que tomar la decisión de educación especial u ordinaria en un tiempo muy reducido y con pocos recursos. La inclusión temprana y la transición progresiva nos da más tiempo y recorrido para acertar en la decisión del camino más adecuado, como veremos a continuación.

\section{V.- Quinta opción: \\ Diferentes caminos de inclusión.}

Esta es una opción abierta en nuestra propuesta y en permanente revisión, ya que pensamos que cada vez más la principal opción para la inmensa mayoría será la atención a la diversidad en la educación ordinaria. Pero, por el momento, dadas las condiciones reales de nuestros centros en Ecuador, presentamos estas opciones, que, por otro lado, las contempla el Ministerio de Educación. Veamos:

\section{Primer camino: la inclusión en la educación escolarizada or-} dinaria, o lo que se conoce como "educación inclusiva". Este es el camino principal de inclusión sin lugar a dudas a nivel mundial, especialmente desde la Conferencia Mundial de la ONU en Salamanca en 1994, y también queremos que lo sea en nuestra propuesta de atención a la diversidad. Está contemplado en la LOEI, en el Reglamento General de la LOEI y en la Ley Orgánica de Discapacidades ${ }^{7}$. Se aborda de manera concreta en el Acuerdo Ministerial 295-13, documento normativo del Ministerio de Educación. En el capítulo III desde el artículo 11 al 19 se especifican las indicaciones al respecto, la descripción, los objetivos, las 
funciones, talento humano, equipo multidisciplinario, la propuesta curricular y la evaluación.

Sin embargo, hay que reconocer que no todas las condiciones que describe el documento se dan en nuestros centros, sobre todo en talento humano necesario y pedagogos de apoyo, y, por consiguiente, no es fácil llevar a cabo las adecuaciones de la propuesta curricular. Se han hecho y se están haciendo grandes esfuerzos desde el Ministerio de Educación y sus distritos, y desde nuestros centros de Fe y Alegría. Y podemos decir con cierto orgullo, que nunca en la historia de la Educación en Ecuador ha habido tantos estudiantes con diversidad funcional en nuestros centros ordinarios tanto fiscales como de Fe y Alegría. La propia propuesta desde edades tempranas y de forma procesual pretende empezar el desarrollo de los estudiantes con diversidad funcional lo antes posible, para que la transición a la escuela ordinaria se dé en las mejores condiciones, y sea la principal opción para la mayoría de ellos.

Pero la realidad es que aun así, nos llegan estudiantes con determinada diversidad funcional que, por el momento, "no son susceptibles de inclusión" en la escuela Ordinaria, según afirma el Acuerdo Ministerial 295-13 en su artículo 4, y buscamos otro camino de inclusión en la escuela Especializada. Todos sabemos que iremos dando pasos en la inclusión educativa ordinaria a la mayoría de los estudiantes, y que, a futuro, sólo los que tengan determinadas características o "limitaciones funcionales intensas", como define la OMS, estarán en centros especializados. Por eso sigue estando vigente el segundo camino.

\section{Segundo camino: la inclusión en Instituciones de Educación}

Especializada. También está apoyada por diferentes documentos y, especialmente por el último Acuerdo Ministerial 295-13. Desde el principio, arts. 3-10, el documento aborda los pormenores de este camino de inclusión para "los estudiantes con necesidades educativas especiales asociadas a discapacidad, no susceptibles de inclusión", dejando esta decisión a criterio de las UDAls, (Unidad Distrital de Apoyo a la Inclusión), como una de sus funciones, y al equipo multidisciplinario de alguna Institución de Educación Especializada. A día de hoy sólo algunos tipos de diversidad funcional, que solíamos denominar como severa, los 
orientamos por este camino: multidiscapacidad, sordoceguera, parálisis cerebral, etc. También los lineamientos de la Dirección Nacional de Educación Especial e Inclusiva de 2015 para las IEE apoyan este camino y presenta los posibles beneficiarios en el apartado 3 .

El dilema se puede presentar con algunos estudiantes que están en el límite de lo que decíamos discapacidad moderada y severa, que además son de inclusión tardía, como algunos estudiantes con síndrome de Down o discapacidad intelectual. En realidad el criterio de "no susceptibles de inclusión" es muy general y difícil de delimitar en algunos casos. Será necesaria una reflexión más profunda. Hasta ciertos padres y madres nos comentan que su hija o hijo no se siente a gusto en la escuela ordinaria, que en la práctica no está incluido, que lo marginan... y que prefiere el camino de la educación especial. Sabemos también que algunos de estos estudiantes que están en Instituciones especializadas muy pronto estarán incluidos en la escuela ordinaria, cuando se cumplan las condiciones necesarias.

Pensamos que, de momento, no se reúnen todas las condiciones para que algunos estudiantes con diversidad funcional se incluyan debidamente en las aulas de educación ordinaria. No se trata sólo de acceso y permanencia, sino de propiciar una verdadera participación y aprendizaje, y, para ello hace falta renovar la cultura, las políticas y las prácticas educativas de los centros.

Tercer camino: la inclusión en un aula de Educación Especializada, dentro de la escuela ordinaria. Este es un camino que está absolutamente abierto a la reflexión y al diálogo, y está siendo objeto de nuestra práctica de "investigación y acción participativa". En realidad sería una variante del camino anterior, porque de hecho es educación especializada. $O$, si se prefiere, puede considerarse un camino medio entre los dos anteriores. Ha tenido, y tiene, sus defensores y detractores; pero más allá de las posibles tendencias y corrientes, esta opción nace de una necesidad claramente sentida en nuestros contextos: No hay una Institución de Educación Especializada cerca de muchos de nuestros centros que pueda ser alternativa a la educación ordinaria. ¿Qué hacer entonces con estos estudiantes que "no son susceptibles de inclusión" en la escuela ordinaria y no tienen instituciones especializadas 
a su alcance? Como ocurre a veces, la necesidad va delante de la teoría y nos mueve a buscar alternativas posibles para dar respuesta a esas necesidades. Este ha sido uno de estos casos.

Lo que pensamos fue crear un Aula de Educación Especializada o Funcional, con el mismo enfoque ecológico funcional que el de las instituciones especializadas, y organizarla como un paralelo más del nivel de educación ordinaria. Suele iniciar en educación Inicial y sería el paralelo " $\mathrm{C}$ " de "Educación Inicial 'especializada' o funcional". Al año siguiente transitan a "Primero de EGB especial o funcional", luego a segundo, etc, de la misma manera que los paralelos de educación ordinaria. Si este grupo estuviera en una IEE, también lo haría de una forma semejante. El MINEDUC propone en los Lineamientos de 2015 el apellido "funcional". Podemos incluso plantearnos la denominación y cambiar el apellido, o sencillamente quitar cualquier apellido, que de hecho sólo está a nivel administrativo, y así evitar una etiqueta más sobre ellos. En todo caso los estudiantes van a convivir en el día a día con un grupo de personas iguales pero con diferente funcionalidad. Son grupos pequeños, según el tipo de diversidad funcional, y en muchos aspectos de la vida escolar coinciden con los de su misma edad, pero con el enfoque ecológico funcional.

Como venimos diciendo, es un camino abierto, que puede ser una vía intermedia entre una educación Especializada "fuera" de la ordinaria (físicamente separada, en otra institución), y otra educación Especializada "dentro" y "a la par" de las aulas de educación ordinaria. En todo caso nos toca seguir reflexionando sobre nuestras prácticas inclusivas y especialmente en determinados contextos.

\section{VI.- Sexta opción: Inclusión Educativa con calidad y recursos suficientes.}

Esta es una opción y también un deseo, que en muchos casos hemos podido cumplir con ayuda de los proyectos de Fe y Alegría y gracias al acuerdo con el Ministerio de Educación y sus distritos; pero lo 
proponemos como algo necesario que no se debe demorar más en todo el país, entre otras cosas porque el marco legal ya lo contempla. Para que una educación sea de calidad pensamos que debería cumplir con algunas premisas o condiciones importantes, como estas:

- Que no sea masificada y se respete la cantidad de estudiantes por aula, y según el tipo de discapacidad, cosa que está bastante clara en documentos normativos actuales (AM 295-13, art. 13).

- Que sea una inclusión bien planificada como el resto de las prácticas educativas, y no sólo para cumplir el dos por ciento obligatorio.

- Que cuente con suficientes profesionales bien formados en los conocimientos necesarios de Educación Especial e Inclusiva. Nos referimos a todos los docentes especialmente, pero también a los equipos de los DECEs o BEI s ${ }^{8}$, y a los equipos de las UDAIS.

- Que se disponga de los especialistas adecuados a cada discapacidad (equipo multidisciplinario): intérpretes y modelos lingüísticos para los sordos, guías para los no videntes, apoyo pedagógico para la discapacidad intelectual, etc... y así se pueda responder a las necesidades reales de los estudiantes de forma eficaz.

Algunas de estas pautas ya se están haciendo, pero no estamos seguros de que llegue a la mayoría de los centros y a todos los lugares de la misma manera y con el tiempo y recursos necesarios. Sabemos que es una tarea muy grande y amplia, y que necesita recursos humanos importantes, pero no podemos detenernos ni retroceder en este camino, sencillamente porque se trata de respetar los derechos de las personas y los acuerdos establecidos.

Al hilo de esta opción será de gran ayuda la confirmación y desarrollo de modelos nacionales necesarios de Educación Especializada e Inclusiva, que sirvan de marco general para todos, y nos den para los DECE. 
los principios y pautas necesarias que no deben faltar en ningún centro. Esto podrá dotar a todas las instituciones de los mismos recursos y oportunidades conforme al derecho común 9

\section{VII.- Séptima opción: \\ Transición a la Vida Adulta y Laboral también en la educación ordinaria.}

Sabemos que es un reto muy grande para la escuela ordinaria, que todavía está dando sus primeros pasos en la educación inclusiva; pero, por muy bien que lo hagamos en los años de escolarización, nuestro trabajo quedaría incompleto si al llegar a la juventud no hemos preparado el paso a la vida adulta y laboral. Tendrá un buen título de EGB en décimo, incluso en Bachillerato, pero su proceso de vida quedaría inconcluso. En algunas instituciones de Educación Especializada con enfoque ecológico funcional es un componente muy importante e imprescindible desde hace unos años, y aun así sabemos que no siempre se realiza con todo el rigor necesario.

Podemos resumirlo de la siguiente manera: La transición a la vida adulta y laboral también llamada inclusión sociolaboral, es el paraguas general que cubre toda la incorporación de un estudiante a la vida adulta, y dentro de ella está el mundo laboral. Para todo ello es necesario preparar al estudiante en dos direcciones u objetivos: Uno, prepararlo en aquellas habilidades que le abran la puerta a la vida en sociedad o al mundo adulto, $y$, dos, formarlo en habilidades y competencias laborales concretas para un trabajo posible.

9 Algunos de ellos ya están muy avanzados y también Fe y Alegría está colaborando directamente, como son: el Modelo de Gestión y Atención para Instituciones de Educación Especial, y el Modelo Bilingüe-Bicultural para Personas Sordas. Sería también muy necesario un modelo bien articulado de Educación Inclusiva en la escuela ordinaria y desde edades tempranas. 
Todo ello se puede articular con un verdadero Programa de Transición, o planificando un Proyecto de Vida. Pensamos que algunas pautas pueden ser estas:

Desarrollo de habilidades para la vida. Desde el principio de la EGB se nos plantea el reto de ir reforzando el desarrollo de habilidades para la vida también en la educación ordinaria con estudiantes con diversidad funcional. Se trata de reforzar no sólo las destrezas del currículo, sino además, y también a la vez, esas habilidades necesarias para vivir en sociedad: pensamos en las habilidades de comunicación, de convivencia, orientación y movilidad, autonomía y autodeterminación, en los diferentes ámbitos donde se mueve una persona. Todo ello habría que incluirlo en las planificaciones que atienden a la diversidad del grupo. Y se puede hacer desde temprana edad. Cabe la posibilidad de adaptar el currículo para llevarlo en una dirección más funcional y práctica, y que el aprendizaje se haga cada vez más con "experiencias de aprendizaje".

Elaboración del Perfil Vocacional. Especialmente desde los 10-12 años conviene ir elaborando el Perfil Vocacional. Es un instrumento vivo, en construcción. Se comienza con diferentes instrumentos de valoración funcional, pero se va construyendo cada año según se observen sus capacidades, preferencias y la experiencia de posibles prácticas prelaborales. Este es un elemento relativamente nuevo en la escuela ordinaria que hay que incorporar en los próximos años.

\section{Valoración de opciones de transición a la vida adulta y labo-} ral. A medida que vayan creciendo, con el apoyo del equipo colaborativo, $\mathrm{DECE}$ o $\mathrm{BEl}$ y familia, hemos de ir ofreciendo algunas opciones de transición, según nos diga el perfil vocacional y las preferencias del estudiante. Una opción puede ser: Continuar estudios, Básica Superior, Bachillerato, Universidad, Cursos técnicos... Y otra opción: Preparación a la transición más inmediata a la vida laboral. Con algunos estudiantes estas opciones no serán claras desde el principio, y conviene que se vayan desarrollando las dos simultáneamente.

Transición a la vida laboral. La transición más inmediata a la vida laboral debería seguir un proceso con los siguientes pasos: 
1.- Continuar con la orientación laboral o vocacional e ir definiendo su perfil vocacional, esto es: Guiar, orientar, acompañar al estudiante para que vaya eligiendo una profesión.

2.- Un segundo paso sería la formación laboral, esto es, la iniciación, práctica y ejercicio de una profesión.

3.- Y el tercer paso sería ya acceder a la ubicación y contrato laboral en regla, de manera más estable.

Hará falta una reflexión importante de toda la comunidad intelectual, y un buen apoyo con recursos y alianzas para que esto se lleve a cabo también en la educación ordinaria.

\section{A Modo de Conclusión y Retos}

Pensamos que con estas opciones se puede construir y llevar a cabo una propuesta de inclusión adecuada. En verdad se han dado pasos maravillosos en todo el mundo para llegar a dotar de igualdad de oportunidades a este colectivo olvidado durante muchos años. Ahora bien, eso no significa que todas las opciones estén desarrollándose adecuadamente, y ni siquiera algunas cuentan con el apoyo de todos. Podemos hacer la siguiente distinción y reflexión final sobre las opciones que hemos tratado:

\section{Hay opciones que están abiertas a la reflexión y al diálogo, y nos plantean retos e interrogantes muy importantes.}

- Este es el caso de la primera opción, por el Enfoque de Atención a la Diversidad. La realidad parece ser que todavía se sigue con el modelo integrador buscando atender las limitaciones de ciertos estudiantes con algún tipo de merma en el aprendizaje, y no se tiene la mirada amplia de atender la diversidad del grupo, o de optimizar los recursos y potencialidades de cada estudiante (García-García et al., 2013). 
-También nos plantea un gran reto e interrogantes la quinta opción: "Diferentes caminos de inclusión, en la educación ordinaria y también en educación especializada". Todos tenemos claro que la educación ordinaria ha de ser cada vez más receptora de estudiantes con diversidad funcional. Muchos estudiantes que ahora tenemos no estaban hace unos pocos años. Pero, por el momento, que conviven los dos caminos, el tema central de esta opción sería: cuáles son los estudiantes que son susceptibles de inclusión en la educación ordinaria. No hay un acuerdo aún cerrado al respecto. Además nos plantea algo más serio y más de fondo: También las instituciones de educación especializada (IEE) son un camino de inclusión educativa.

\section{Hay opciones que todos aceptamos, pero no se están po- niendo en marcha.}

-Es el caso de la necesaria calidad de la educación y de los modelos de gestión. En realidad todos pensamos que es un tema económico, pero también de tiempo. Lleva tiempo poner en marcha los procesos de inclusión con todas las diversidades funcionales. Lleva tiempo preparar a la ciudadanía y lleva tiempo preparar a los centros y a los profesionales, la cultura, políticas y prácticas educativas.

-Creemos que otro tanto ocurre con la inclusión temprana de personas con diversidad funcional. Ciertamente es muy costoso preparar centros y profesionales para la inclusión temprana, pero hace falta tiempo también para que la ciudadanía lo vea como una necesidad, y busque y decida este servicio.

-Un gran reto sería la preparación de la transición a la vida adulta y laboral también en la educación ordinaria. Todos sabemos que lo tenemos que realizar necesariamente, y también que hemos de prepararnos para ello, especialmente en la escuela ordinaria.

Hay opciones que todos aceptamos y se van cumpliendo en mayor o menor medida. Creemos que una de ellas es el Enfoque Ecológico Funcional. Al menos en teoría, casi todos han realizado cursos y talleres al respecto. Ahora bien, otra cosa es que se aplique y se desarrolle a cabalidad en los centros. 


\section{Bibliografía}

Echeita, G. y Domínguez, A. (2011). Educación inclusiva. Argumentos, caminos y encrucijadas, Aula, vol. 17, pp. 23-35.

Echeita, G. y Ainscow, M. (2011). La educación inclusiva como derecho. Marco de referencia y pautas de acción para el desarrollo de una revolución pendiente, Tejuelo, vol. 12, pp. 26-46.

Núñez, M. y otros (2014). Enfoques de atención a la diversidad, estrategias de aprendizaje y motivación en educación secundaria. Perfiles Educativos, vol. XXXVI, núm. 145, pp. 65-80. Instituto de Investigaciones sobre la Universidad y la Educación. Distrito Federal, México. Consultado en : http://www.redalyc.org/ pdf/132/13231362005.pdf

Pastor, C. y otros (2013). Pautas sobre el Diseño Universal para el Aprendizaje (DUA) Texto Completo (Versión 2.0). Universidad Complutense de Madrid.

Educacantabria (2017) Atención a la diversidad Consultado en: https:// www.educantabria.es/planes/atencion-a-la-diversidad.html.

Federación Internacional de Fe y Alegría (2014). Programa de Informática Educativa. Guía Conceptual Módulo I. "Comunidad Educativa Inclusiva". FIFYA.

Gallegos, Miriam y De la Cadena, Yanira (2014). Guía Conceptual Módulo II "Barreras para la Inclusión Educativa de estudiantes con discapacidad". FIFYA.

Aguilera Bastidas, Rosaicela Ivon (2015). Guía conceptual Módulo III "Inclusión y TIC". FIFYA.

Yépez, M. y otros; (2011-13). Programa de capacitación a docentes y familias para el desarrollo de la Educación Especial. Módulo I: Diseño curricular con enfoque ecológico funcional en la educación de personas con discapacidad. Fe y Alegría Ecuador, BID, UPS Ecuador. 
MINEDUC (2013) Guía de trabajo- adaptaciones curriculares para educación especial e inclusiva. Pp. 22-53.

MINEDUC (2014) Currículo de educación inicial.

OMS y Unicef. (2013). El desarrollo del niño en la primera infancia y la discapacidad: un documento de debate. Consultado en: https:// www.unicef.org/earlychildhood/files/ECDD_SPANISH-FINAL_ (low_res).pdf).

Gallegos, M. y otros (2004). Plan Nacional de formación laboral. MINEDUC. DNEE.

Jaramillo, A. y López, R. (2016) Guía y Herramientas de inclusión socio laboral para personas con discapacidad. CBM para América Latina y el Caribe. 